\title{
Genetic Algorithms for Predicting DISCRETE SEQUENCES
}

\author{
KREMLJAK, Z.
}

Abstract: The paper presents the statistical method which uses genetic algorithms for predicting a discrete sequence. Learning from experience to predict discrete sequences is a fundamental problem in machine learning with many applications. Most of the modelling algorithms reported in the literature focus on either determining the order or estimating the model parameters. The sequence imposes an order on the observations that must be preserved when training models and making predictions. Predicting the next value is based on a specific number of preceding values (input sequence) and statistics. Subject to statistics, the continuation of similar previous cases indicated the occurrence of different values. The examination thereof by applicable weights leads to successful forecasting. Weights are generated through genetic algorithms. The paper presents a new hybrid method for predicting discrete chronological sequences combining the Markov model and genetic algorithm to optimise its effect. Experiments with various data sets verify its performance.

Key words: Artificial intelligence, Genetic algorithms, Predicting discrete sequences, Forecast
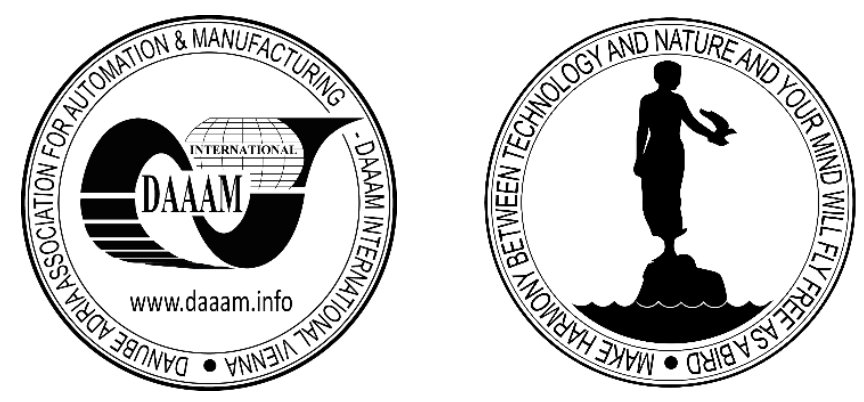

Authors' data: Asst. Prof. Dr. Sc. Kremljak, Z[vonko], Private researcher, Hmelina 14, 2360 Radlje ob Dravi, Slovenia, EU, zvonko.kremljak@s5.net

This Publication has to be referred as: Kremljak, Z[vonko] (2021). Genetic Algorithms for Predicting Discrete Sequences, Chapter 04 in DAAAM International Scientific Book 2021, pp.039-050, B. Katalinic (Ed.), Published by DAAAM International, ISBN 978-3-902734-31-0, ISSN 1726-9687, Vienna, Austria

DOI: $10.2507 /$ daaam.scibook.2021.04 


\section{Introduction}

Predicting discrete sequences is an issue plaguing computing, mechanical engineering, economy and other areas. A concrete case of a discrete sequence, in which only a specific number of different symbols set in advance can appear in a sequence, is extremely complex in and of itself and is used for shortening texts and managing computer memory. Sequence also play an important role in the acquisition of human skills.

For a detailed overview of various sequence learning models, refer to He (2011). Various sequence-related neural network models have also been developed, such as periodic re-transfer networks, which are frequently used for sequence prediction problems. Hidden Markov models (HMMs) are also suitable for problems associated with predicting discrete sequences (Bengio, 1996). Evolutionary accounts are another alternative whose general nature can be used in all types of problems associated with learning sequences.

This paper shall present a new hybrid method for predicting discrete sequences combining the Markov model and genetic algorithms to optimise its effect and fast computer-based decision-making (Goldberg, 1989) on the basis of mapping the problem domain from mathematical code into genetic code and chromosomes.

\section{Prediction Method}

The discrete sequence predictor is a tool which can predict the following symbols in the sequence if preceding observations of value are at its disposal. Our aim has been to create a predictor which would be able to predict the following value of the sequence as accurately as possible by relying on a number of preceding values (Brucker et al., 1999). If $N$ preceding $X_{1}, \ldots, X_{K}, \ldots, X_{N}$ values have been registered, $X_{N+1}$ can be predicted subject to the preceding $1,2,3, \ldots N$ values.

Without restricting the general nature of the problem, it can be predicted that the most likely symbols to occur in the sequence are the numbers $0, \ldots, K, \ldots, k-1$, whereby $k$ stands for the number of different symbols in the sequence.

$P_{i}{ }^{j}(X, N)$ stands for the probability that in an $i$-based sequence, the most recent preceding values $X_{N-i}, \ldots, X_{K}, \ldots, X_{N}$, and $j$ shall follow (meaning that $X_{N+1}=j$ ).

Since the optimal number of preceding values on which a prediction should be based is usually not known, a weighted sum of all predictions based on $1,2, \ldots, K, \ldots$, $N$ preceding values will be used. With this in mind, the probability of the next value being $j$ can be calculated as follows:

$$
P^{j}(X, N)=\sum_{i=1}^{N} W_{i} \times P_{i}^{j}(X, N)
$$

whereby $W_{i}$ is the weight of predictions based on the value $i$ that symbol $j$ is going to occur in the following sequence.

Subject to the two sub-tasks that will be performed, the exercise data have been divided into two parts: 
- set of data for construction statistics,

- set of data for calculating the weights.

As far as the first set of data is concerned, probabilities $P_{i}^{j}(X, N), j=0,1, \ldots, K$, $\ldots, k-1$ are calculated for all possible sequences of $N$-long symbols which have occurred in this part of the sequence. The calculation method is based on building a log probability for possible sequences of maximum $N$ lengths as described by Laird and Saul (1994).

Subsequently, a genetic algorithm will be used to find applicable weights. The foreseen following value by using the applicable weight vector is:

$$
X_{N+1}=j, \text { whereby } P^{j}(X, N)=\max _{l=0}^{k-1} P^{l}(X, N)
$$

Weights have been developed for precise predictions of symbols in the second set of data (Demšar, 2008).

\section{Heuristics}

Many optimization problems, whether of a practical or theoretical in nature, are so demanding that precise algorithms take too much time to solve (Sivanandam \& Deepa, 2008). Thus, solving with heuristic methods is possible, with which we sacrifice the assurance that we will find the optimal solution, but we get a relatively good solution in a much shorter time. We use neural networks, genetic algorithms, ant colonies (Ioannidou et al., 2011; Wikipedia ${ }^{1}$, 2020) ..., unlike classical computational methods that execute program commands sequentially. These methods perform operations on potential solutions iteratively until the criterion function reaches the optimum. Evolutionary algorithms (work with coded variables) are used, as well as simultaneous search of a set of solutions and evaluation with a capability function (suitability - adequacy of the solution) (Wikipedia ${ }^{2}, 2020$; Wikipedia $\left.^{3}, 2020\right)$. The use of genetic functions - probability operators (selection, crossing, mutation) details of the problem to be solved and probability operators - suitable for nonlinear problems.

\section{Genetic Algorithms}

As the most widespread type of evolution algorithms, genetic algorithms (GA) have become a very popular means of heuristically resolving optimisation-related issues. GA have proven extremely powerful particularly in cases when solutions from a wide scope of examination are required. Hitherto, GA have resolved nondeterministic problems (Chambers, 2000; Karova et al., 2008). Solutions have not been sought in pre-determined ways and an array thereof has been handled simultaneously which has proven particularly appropriate for problems faced in complex production and business processes. Instead of certainty, order and logical deductions, we are faced with randomisation, evolution and self-organisation (Chen \& Hao, 2018). Evolution algorithms have been present since the early 1960s. 
So far, genetic algorithms have proven the best and most robust evolution algorithms who seek approximate solutions to challenging problems through principles of inheritance subject to Darwin's paradigm. Genetic algorithms operate on an array of possible solutions, whereby other heuristic algorithms in their iterations apply only one solution (Brusco, 2015). Another important difference also lies in the fact that GA are stochastic and not deterministic algorithms (Bäck, 1996). A proper coding of chromosomes is most likely the most important factor for the performance of GA. They have proven extremely useful in solving complex production problems where traditional methods fail to work and can make key contributions to making strategic decisions (Štumberger, 2011; Gocken \& Yaktubay, 2019).

More important than an accurately predicted condition, which can be too late, is the way we simulate business conduct as a whole. In particular, we are on the look-out for reasons for decisions on the one hand and opportunities which provide us with added value at the same price on the other. By only using one modelling and singledirection-oriented thought process, we can quickly miss the actual reason or opportunity. Modelling or looking for various solutions by applying evolution methods can provide us with a completely new view of the existing condition and the reason for poor performance (Man et al., 1999). Future research is going to focus on complementing the system model for applying further evolution methods (Tabu search, PSO, etc.). Genetic functions are: selection, crossover, and mutation (Aridolan, 2020). These functions recombine existing individuals into new ones, representing new solutions. We need to determine in advance the size of the population, the number of parents to choose from, the degree of mutation, the performance evaluation (fitness) function etc. (Zhang \& Zhang, 2020; Rosyid, 2018).

\subsection{Crossover}

We used two parents and two children and one position cross, with a predetermined cross position. For one position cross, the rule is to keep the rules of chromosome assembly, thus avoiding rapid convergences, after a possible mutation (Tab. 1).

Parent 1
\begin{tabular}{|c|c|c|c|c|}
\hline 0,72 & 0,84 & 0,44 & 0,84 & 0,84 \\
\hline 0 & 0,56 & 0.84 & 0 & 0,44 \\
\hline 0 & 0,28 & 0 & 0,44 & 0,16 \\
\hline 0,84 & 0 & 0,56 & 1 & 0,56 \\
\hline 0,28 & 0,44 & 0 & 0,16 & 0,28 \\
\hline
\end{tabular}

\begin{tabular}{l|c|c|c|c|} 
Parent 2 \\
\hline 0,84 & 0.44 & 0,16 & 0,72 & 0,72 \\
\hline 0 & 0,44 & 0,72 & 0 & 0,16 \\
\hline 0 & 0,16 & 0,44 & 0.28 & 0,28 \\
\hline 0,72 & 0 & 0,44 & 0,48 & 1 \\
\hline 0 & 0,28 & 0 & 0,28 & 0,16 \\
\hline
\end{tabular}


Child 1

\begin{tabular}{|c|c|c|c|c|}
\hline 0,72 & 0,84 & 0,44 & 0,84 & 0,84 \\
\hline 0 & 0,56 & 0,84 & 0 & 0,44 \\
\hline 0 & 0,16 & 0,44 & 0,28 & 0,28 \\
\hline 0,72 & 0 & 0,44 & 0,84 & 1 \\
\hline 0 & 0,28 & 0 & 0,28 & 0,16 \\
\hline
\end{tabular}

Child 2

\begin{tabular}{|c|c|c|c|c|}
\hline 0,84 & 0,44 & 0,16 & 0,72 & 0,72 \\
\hline 0 & 0,44 & 0,72 & 0 & 0,16 \\
\hline 0 & 0,28 & 0 & 0,44 & 0,16 \\
\hline 0,84 & 0 & 0,56 & 1 & 0,56 \\
\hline 0,28 & 0,44 & 0 & 0,16 & 0,28 \\
\hline
\end{tabular}

Tab. 1. Example of one position crossing of parent 1 and parent 2.

\subsection{Mutation}

The mutation used is a derivative of the random bit rotation mutation. In random rotation, one or more randomly selected bits in the chromosome are selected. In our case, all genes in a randomly selected chromosome line. We preserve the zeros that are conditioned by the first rule of chromosome conservation. We maintain the normalization of the mutation factor, via the interface. The mutation factor is set by the user via the user interface (Tab. 2).

Child 1

\begin{tabular}{|c|c|c|c|c|}
\hline 0.72 & 0.84 & 0.44 & 0.84 & 0.84 \\
\hline 0 & 0.56 & 0.84 & 0 & 044 \\
\hline 0 & 0,16 & 0,44 & 0,28 & 0,28 \\
\hline 0,56 & 1 & 0,84 & 0,72 & 0,16 \\
\hline 0 & 0,28 & 0 & 0,28 & 0,16 \\
\hline
\end{tabular}

Tab. 2. Example of a child 1 mutation.

Parameter values are used to test different strategies for selecting gene pairs to be digested. The number of generations that are produced in the evolutionary cycle must be determined at the beginning of the optimization process and the initial value must be large enough to provide an improvement in the variable. Tests show that 50 generations is enough to achieve optimization results in cases of problems of our type. With the stop criterion, it is possible to interrupt the optimization process when the variable becomes almost static over a given successive number of generations or when a previously determined level of function is reached.

The impact of optimization strategies is achieved by the value of parameters that determine the relationships between gene crossover, adaptation and mutation, and the likelihood of each. Most of these parameters can be used to increase the search space and variety of solutions. More likely for mutations, transfection and adaptation, the genetic algorithm tells us to find more possible solutions. 
The "keep the best specimens" parameter is used to increase the speed of the search process, but good solutions may be overlooked. Optimization is divided into several optimization phases (Mernik et al., 2003; Blazewicz et al., 1983). In essence, each gene value is limited by minimum and maximum values, and non-sequential chromosomes are limited by the sum of all genes in one chromosome.

Unfortunately, constraints are rarely defined as a fixed number, and most constraints are defined by terms that define the interval for these constraints. Parameter values are used to test different strategies for selecting gene pairs to be digested. The number of generations that are produced in the evolutionary cycle must be determined at the beginning of the optimization process and the initial value must be large enough to provide an improvement in the variable.

A multi-directional examination has been carried out in space with $\mathrm{N}$-dimensional weight vectors. If we start with a random population of weight vectors and continue to use so-called genetic operators, an improved weight vector is generated. The effect of weight vectors has been evaluated in the second sub-set of experiment data (these data differ from the data used to create a log likelihood). The driving source of evolution is to select more effective (or better adapted) individuals in each generation, meaning that better individuals are more likely to survive through various generations (Habiballa et al., 2021; Cassar et al., 2021).

In our case the genetic algorithm has been designed with the aim of identifying weights of predictions based on a varying number of preceding values (Brezočnik et al., 1990; Weigdong et al. 2014; Math.NET, 2020). General system of GA is shown in Fig. 1.

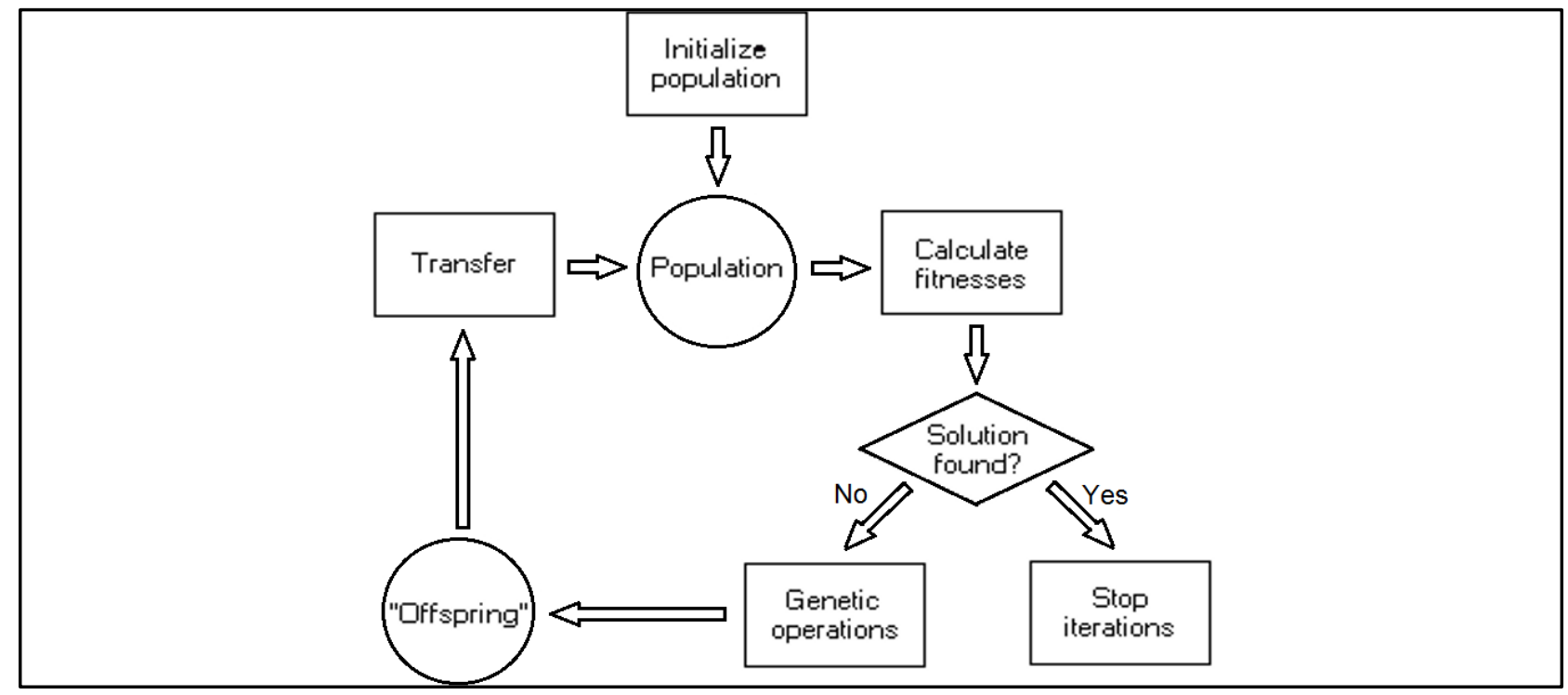

Fig. 1. Optimization with GA (flowchart) - general working scheme.

\section{Statistical Information Maintenance - Associated Data Structure}

A $\log$ likelihood based on the selected part of the sequence has been generated. For each sub-sequence of length $i(i=0, \ldots, K, \ldots, N)$ the path runs from the root of the $\log$ to the node at depth $i$ in the $\log$ (as a rule, the root is at $i=0$ depth). The $j^{\text {th }}$ descendant of the node at depth $i$ corresponds the situation in which symbols $i$ in the 
corresponding sub-sequence $X_{N-1}, X_{K}, X_{N}$ are followed by $X_{N+1}=j$. This node contains a piece of information which constitutes the value of probability $P_{i}^{j}(X, N)$.

For simplification purposes, the log is presented through a binary sequence $(k=2)$ containing symbols 0 and 1 . Let us assume that the sequence is as follows (Demšar, 2008): "101100101011".

Fig. 2 shows the $\log$ likelihood for $N=2$ preceding values. The left descendant of each node corresponds to symbol 0 and the right to symbol 1 . The number of occurrences of the corresponding sub-sequence are marked on each arc. The marked node containing 0.67 corresponds sub-sequence 1 follows by 0 . In this particular subsequence 1 is followed by four 0 and two 1 , as marked on the arcs. The log is built in two steps. The first step constitutes the building of a log structure on the basis of the relevant sequence.

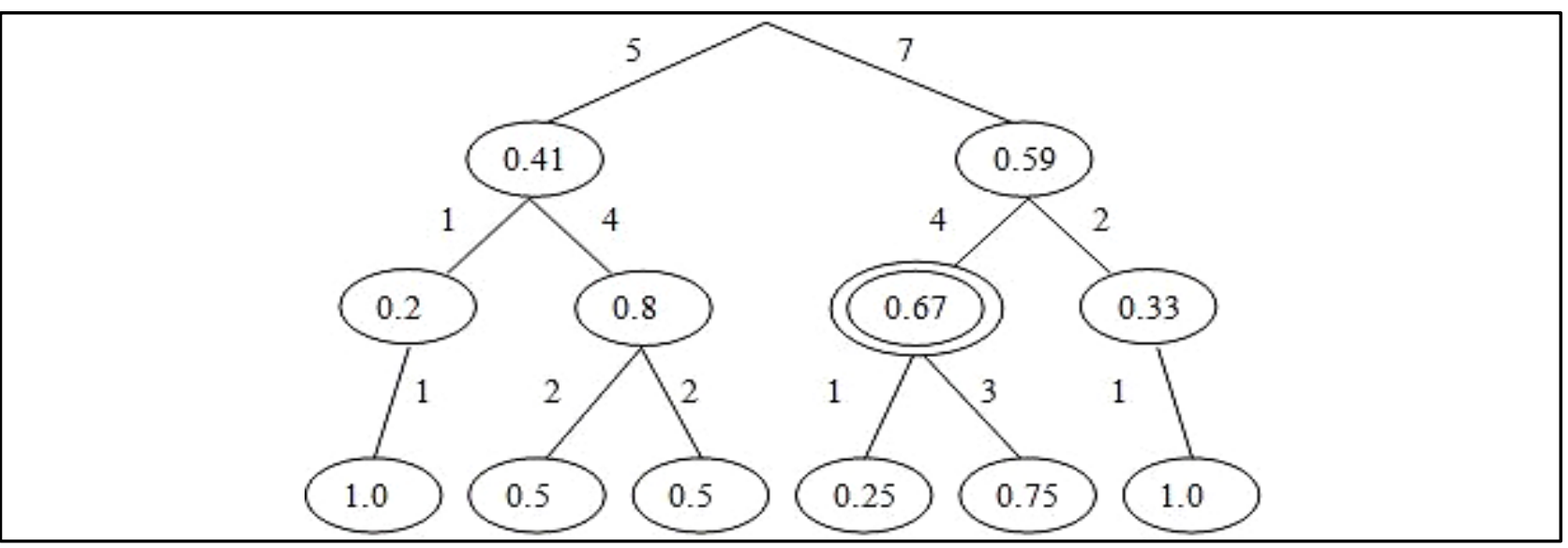

Fig. 2. Log likelihood example.

Where applicable, new nodes are generated dynamically and information associated with the corresponding arcs are upgraded. As soon as the log is completed, when, e.g. all data in the sequence have been considered, probabilities are calculated. Fig. 3 shows convergence of GA course steps.

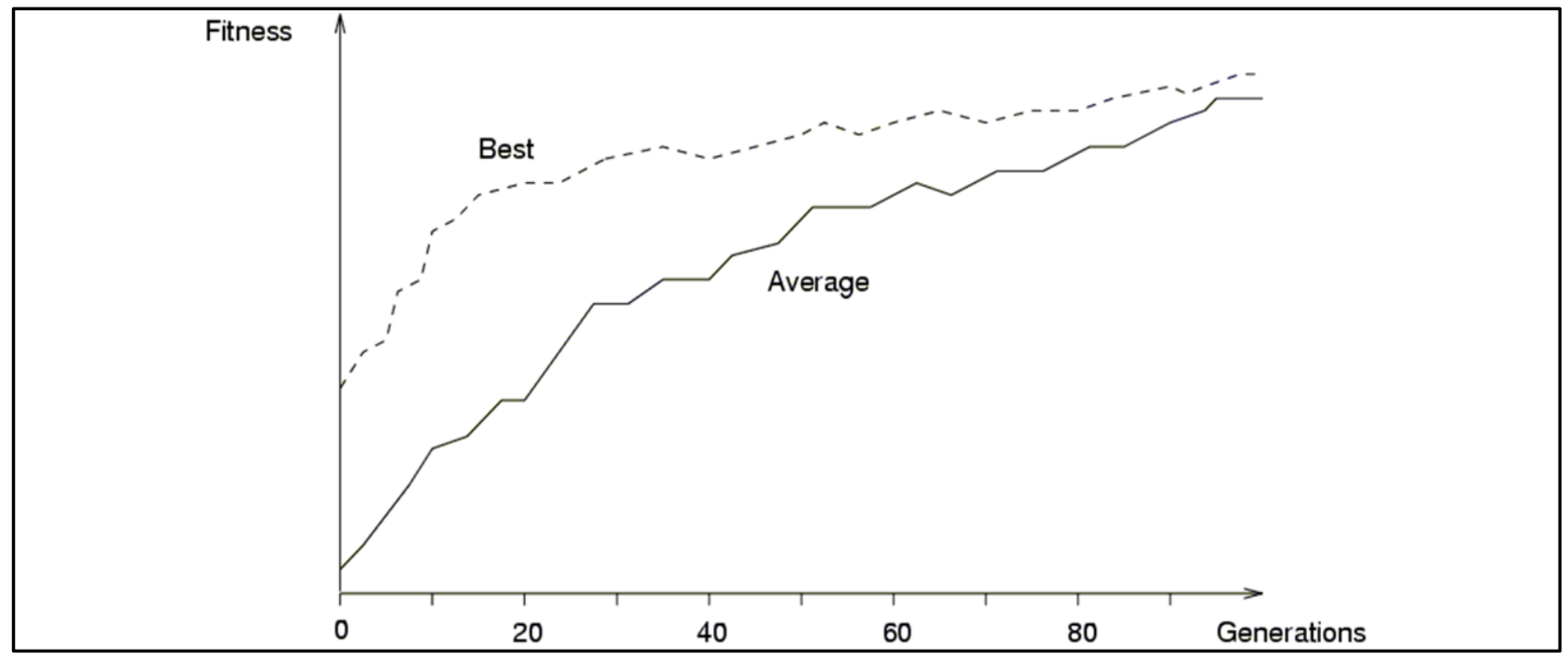

Fig. 3. Example of convergence. 
Let us now consider the possibility that 01 occurs at a later point in the sequence. In that case, the probability of 0 or 1 occurring as the following symbol is 0.5 for both, namely by considering the prediction based on two preceding values. Subject to the preceding value, the probability of 0 occurring is 0.67 , as such 0 will be predicted as the following symbol. If preceding values are not considered, the probability of 1 occurring is 0.59 , as such 1 will be predicted as the following symbol. A weighted sum of these probabilities may lead to more reliable forecasting. Mapping the problem domain from mathematical code (Talbi, 2009) into genetic code is shown in Fig. 4 and the coding chromosomes in Fig. 5.

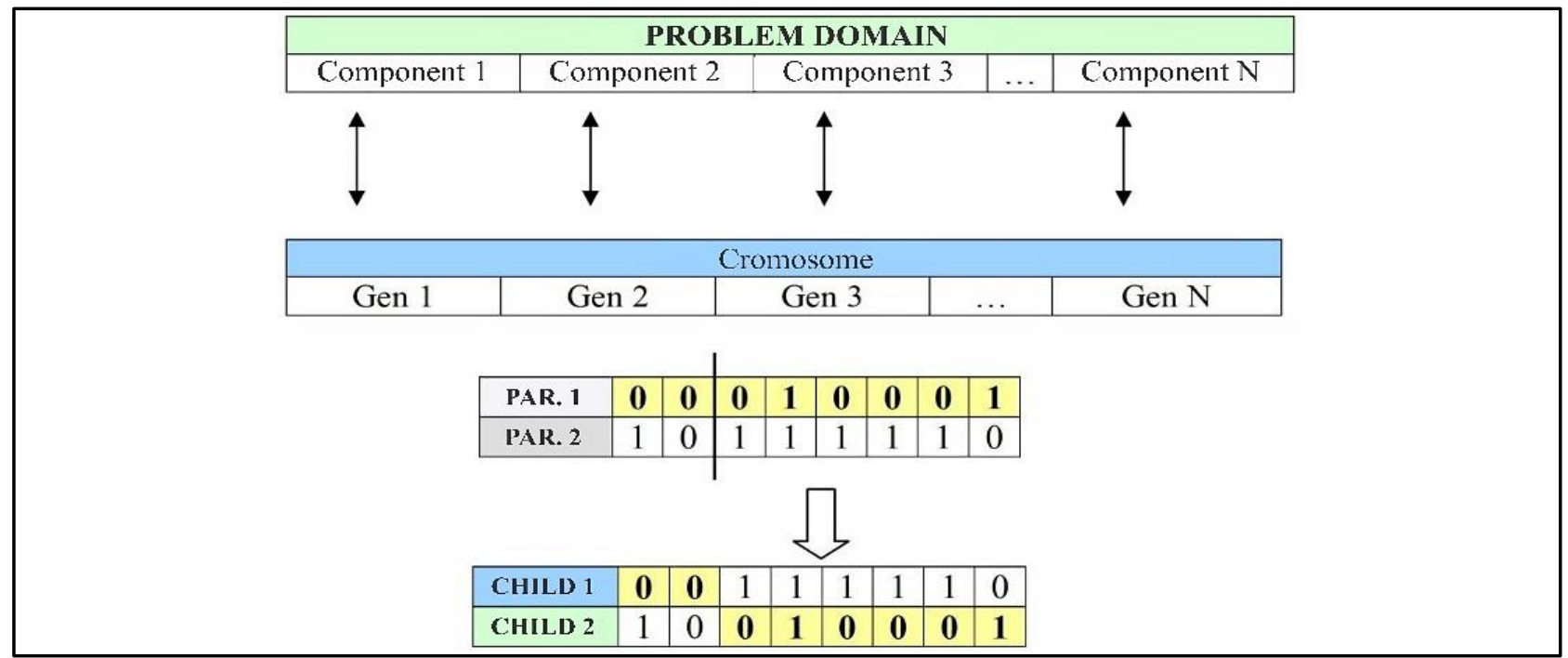

Fig. 4. Mapping the problem domain from mathematical code into genetic code.

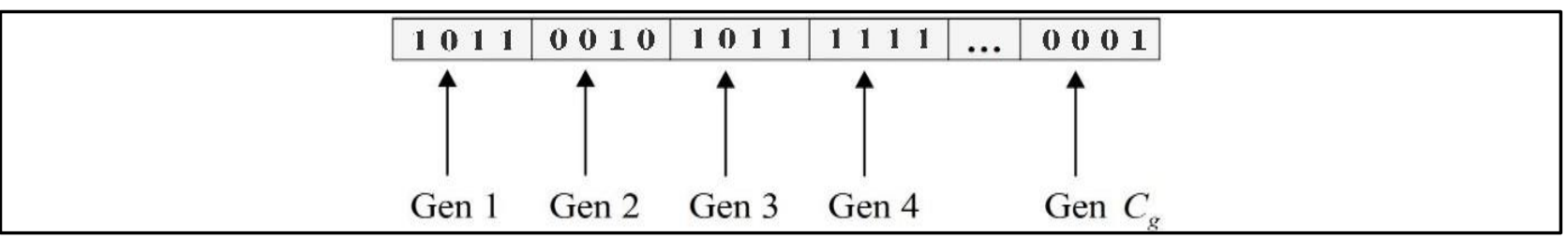

Fig. 5. Coding chromosomes.

\section{Application}

The subject of examinations are binary sequences. A sequence contains 10.000 data bits. The first 9.000 bits were used for training the predictor. This was followed by verifying its level of precision by means of the remaining 1.000 bits. $N=100$ preceding values and data ratios were used for generating the log. It was found that the level of precision of the involved predictors is dependent on the used genetic search operators and the data ratios used for generating the log likelihood and producing weights.

The following three types of crosses were tested (see Tab. 3):

- simple crosses - parents $P_{1}$ and $P_{2}$ and $x$ cross point lead to descendants:

$$
C_{1}=P_{1} \mathrm{~K} P_{1}(x) P_{2}(x+1) \mathrm{K} P_{2}(N) \text { and } C_{2}=P_{2}(1) \mathrm{K}_{2}(x) P_{1}(x+1) \mathrm{K} P_{1}(N),
$$

- medium crosses: the same as simple crosses with the exception of cross point genes:

$$
C_{1}(x)=\alpha \cdot P_{1}(x)+(1-\alpha) \cdot P_{2}(x) \text { and } C_{2}(x)=(1-\alpha) \cdot P_{1}(x)+\alpha \cdot P_{2}(x) \text {, }
$$


- mixed crosses: the same as simple crosses with the exception of cross point genes selected as random number at an interval:

$$
\left[\begin{array}{l}
P_{1}(x)-\alpha\left(P_{2}(x)-P_{1}(x)\right), P_{2}(x) \\
+\alpha\left(P_{2}(x)-P_{1}(x)\right)
\end{array}\right]
$$

\begin{tabular}{|c|c|c|c|c|}
\hline \multirow{2}{*}{$\begin{array}{c}\text { Deviations } \\
(\%)\end{array}$} & \multicolumn{2}{|c|}{ 7_2_1 } & \multicolumn{2}{c|}{$8 \_1 \_1$} \\
\cline { 2 - 5 } & training & test & training & test \\
\hline $\begin{array}{c}\text { Simple } \\
\text { crosses }\end{array}$ & 8.31 & 9.04 & 7.52 & 8.65 \\
\hline $\begin{array}{c}\text { Medium } \\
\text { crosses }\end{array}$ & 8.24 & 8.93 & 7.65 & 8.61 \\
\hline $\begin{array}{c}\text { Mixed } \\
\text { crosses }\end{array}$ & 8.25 & 8.98 & 7.36 & 8.54 \\
\hline
\end{tabular}

Tab. 3. Average deviations for solutions obtained in various experiments.

Tab. 4 contains the number of errors per 100 predictions for involved predictors divided into 10 sets of the genetic algorithm (see Fig. 6, too). Results for two experiment data distributions are illustrated: 7.000 for the log likelihood, 2.000 for weights (7_2_1) and 8.000 for the $\log$ likelihood, 1.000 for weights (8_1_1). The subject of the experiments were binary sequences.

\begin{tabular}{|l|c|}
\hline Population size & 100 \\
\hline Individual & Weighting factor $W$ \\
\hline Gene & Weight $W$ \\
\hline Probability of cross & $9 \%$ \\
\hline Mutation probability & $10 \%$ \\
\hline Selection method & Roulette \\
\hline Maximum number of generations & 50 \\
\hline
\end{tabular}

Tab. 4. Parameter settings for the genetic algorithm.

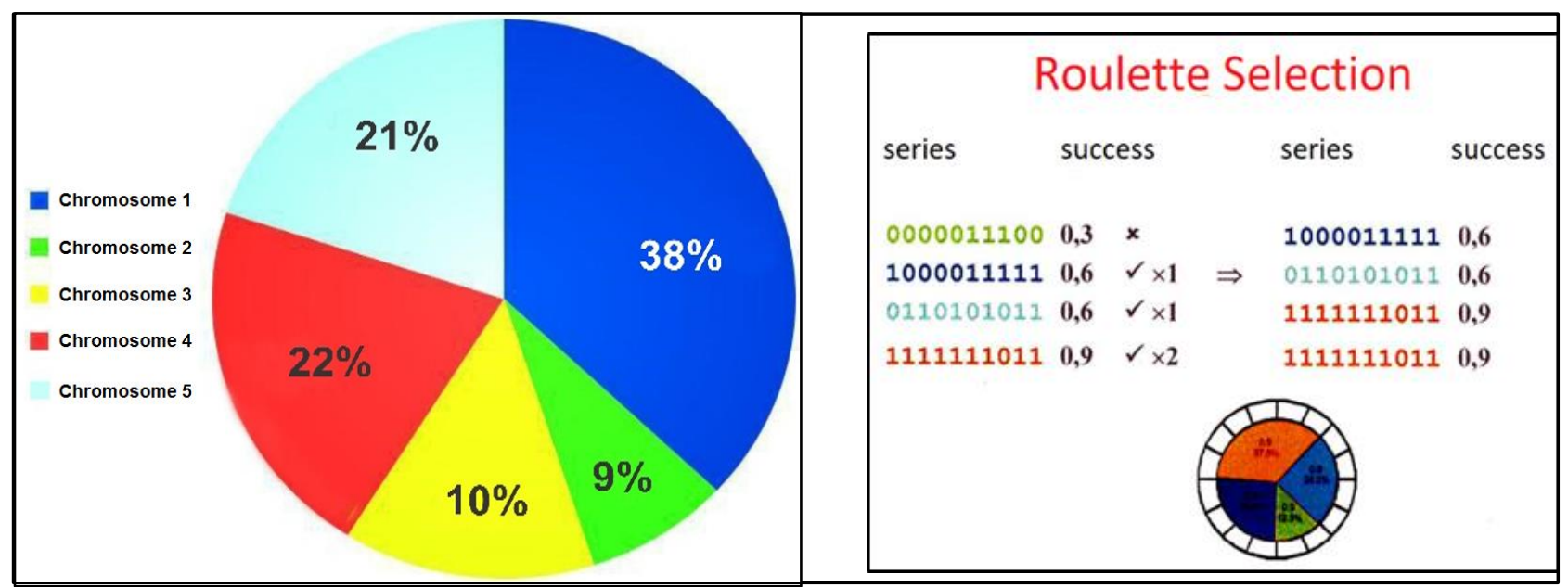

Fig. 6. Roulette example (the probability of chromosome selection is proportional to its success). 


\section{Conclusion}

The genetic algorithm (GA) as a type of evolutionary GA algorithms is primarily used to solve problems where deterministic algorithms are time consuming. GA takes place in several iterations (generations), in which genetic operations are performed on a set of permissible solutions, called a population of chromosomes. The population of genetic diversity is made up of chromosomes whose fitness estimates are diverse enough to prevent rapid convergence of GA into local optimums. The population of flexible genetic diversity, however, is characterized by rapid completion of GA in local optimums, solutions are not good. The choice of selection method, the way of creating and updating the population strongly influences the genetic diversity of the population. We used random roulette, random selection, priority selection, and elitism. The combination of random roulette selection and rapid population growth proved to be the worst. From the results, we learned that elitism proved to be the best choice of the method of selection, where we choose between only twenty percent of the best parents when crossing. However, slow growth has proven to be the most effective way to grow the population. In the latter, the population increases by ten percent of the previous population each generation, which further increases the genetic diversity of the population. In the mode of rapid population growth, we highlighted the problem of the rapid growth of time complexity for each new generation.

Discrete sequence prediction (DSP) is one of the basic learning problems. Predictions from different individuals are usually quite similar, so, this is an evidence that DSP is a fundamental skill in the human learning repertory. It plays an important role in information-theoretic applications, dynamic program optimization, dynamic buffering algorithms, adaptive human-machine interfaces and in detection systems.

It was found that the level of precision of the involved predictors is dependent on the genetic search operators used for generating the log likelihood and producing weights.

The predictors are more precise if the ratios of data used for generating the log are higher. Differences in various types of crosses are extremely small. The involved predictors are highly resistant and can be easily generalised to non-observed test data.

It can be concluded that the presented method provides rather precise predictors for discrete sequences by assuming that sufficient sequences for generating reliable statistics are available.

Advantages of this method are:

- work with coded variables,

- search with the population of solutions,

- use of the performance function,

- application of probability rules.

Heuristic used in the article are accurate and fast enough in making and simulating decisions methods. Successful problem-solving methods do not need to be smart but capable of learning from experience.

Future research plans include the study and use of other predicting methods, extensive testing on real data, their comparison, performance evaluation (accuracy), and appropriate suggestions for application in specific conditions. 
The use of artificial intelligence methods is on the rise and we can anticipate their further expansion in practically all areas of application. The constant growth of the computing power of processors enables the increasingly intensive use of these methods.

\section{References}

AriDolan. An Overview of Artificial Intelligence, available from: http://www.aridolan.com._Accessed: 2020-07-10

Bäck, T. (1996). Evolutionary Algorithms in Theory and Practice: Evolution Strategies, Evolutionary Programming, Genetic Algorithms, Oxford University Press, ISBN 978-0195099713, Oxford

Bengio, Y. (1996). Markovian Models for Sequential Data, Technical Report \#1049, Departement d'Informatique et Recherche Operationnelle, Universite de Montreal, Montreal

Blazewicz, J.; Lenstra, J. K. \& Rinnooy Kan, A. H. G. (1983). Scheduling subject to resource constraints: classification and complexity. Discrete Applied Mathematics, Vol. 5, No. 1, pp. 11-24, ISSN 0166-218X

Brezočnik, Z.; Horvat, B.; Kapus, T. \& Dreo, G. (1990). Simulacija računalniških sistemov in komunikacijskih mrež (Simulation of computer systems and communication networks), University of Maribor, Faculty of Technical Sciences, ISBN 978-86-435-0001-1, Maribor (in Slovenian)

Brucker, P.; Drexl, A.; Mohring, R.; Neumann, K. \& Pesch. E. (1999). Resourceconstrained project scheduling: Notation, classification, models, and methods. European Journal of Operational Research, Vol. 112, No. 1, pp. 3-41, ISSN 0377-2217 Brusco, M. J. (2015). An iterated local search heuristic for cell formation. Computers \& Industrial Engineering, Vol. 90, pp. 292-304, ISSN 0360-8352

Cassar, D. R.; Santos, G. G. \& Zanotto, E. D. (2021). Designing optical glasses by machine learning coupled with a genetic algorithm. Ceramics International, Vol. 47, No. 8, pp. 10555-10564, ISSN 0272-8842

Chambers, L. D. (Ed.) (2000). The Practical Handbook of Genetic Algorithms: Applications, $2^{\text {nd }}$ Edition, Chapman and Hall/CRC, ISBN 978-1584882404, Boca Raton, FL

Chen, W. \& Hao, Y. F. (2018). Genetic algorithm-based design and simulation of manufacturing flow shop scheduling. International Journal of Simulation Modelling, Vol. 17, No. 4, pp. 702-711, ISSN 1726-4529

Demšar, J. (2008). Projektiranje in organizacija informacijskih sistemov - mrežno načrtovanje (Design and organization of information systems - network planning), 33 PPT slides, University of Ljubljana, Faculty of Computer and Information Science, Ljubljana (in Slovenian)

Gocken, T. \& Yaktubay, M. (2019). Comparison of different clustering algorithms via genetic algorithm for VRPTW. International Journal of Simulation Modelling, Vol. 18, No. 4, pp. 574-585, ISSN 1726-4529

Goldberg, D. E. (1989). Genetic Algorithms in Search, Optimization, and Machine Learning, Addison-Wesley Professional, ISBN 978-0-201-15767-3, Boston, MA 
Habiballa, H.; Volna, E. \& Kotyrba, M. (2021). Automated generation of EQ-algebras through genetic algorithms. Mathematics, Vol. 9, No. 8, Article 861, EISSN 22277390

He, H.-B. (2011). Self-Adaptive Systems for Machine Intelligence, John Wiley \& Sons, Inc., ISBN 978-0470343968, Hoboken, New Jersey

Ioannidou, K.; Mertzios, G. B. \& Nikolopoulos, S. D. (2011). The longest path problem has a polynomial solution on interval graphs. Algorithmica, Vol. 61, pp. 320-341, ISSN 0178-4617

Karova, M.; Petkova, J. \& Smarkov, V. (2008). A genetic algorithm for project planning problem, $4^{\text {th }}$ International Scientific Conference: Computer Science 2008, pp. 647-651, ISBN 978-954-580-256-0, Kavala, Greece, September 2008

Laird, P. \& Saul, R. (1994). Discrete sequence prediction and its applications. Machine Learning, Vol. 15, pp. 43-68, ISSN 0885-6125

Man, K. F.; Tang, K. S. \& Kwong, S. (1999). Genetic Algorithms, Springer-Verlag, ISBN 978-1-4471-0577-0, London

Math.NET. Math.net numerics, available from: https://numerics.mathdotnet.com/. Accessed: 2020-07-16

Mernik, M.; Črepinšek, M. \& Žumer, V. (2003). Evolucijski algoritmi (Evolutional algorithms), University of Maribor, Faciluty of Electrical Engineering and Computer Science, ISBN 978-8643505939, Maribor (in Slovenian)

Rosyid, A.; El-Khasawneh, B. \& Alazzam, A. (2018). Genetic and hybrid algorithms for optimization of non-singular 3PRR planar parallel kinematics mechanism for machining application. Robotica, Vol. 36, No. 6, pp. 839-864, ISSN 0263-5747

Sivanandam, S. N. \& Deepa, S. N. (2008). Introduction to Genetic Algorithms, Springer-Verlag, ISBN 978-3-540-73190-0, Berlin

Štumberger, D. (2011). Uporaba genetskega algoritma pri upravljanju programskih projektov (Use of genetic algorithm in software project management), Diploma Thesis, University of Ljubljana, Faculty of Computer and Information Science, Ljubljana (in Slovenian)

Talbi, E.-G. (2009). Metaheuristics: From Design to Implementation, John Wiley \& Sons, Inc., ISBN 978-0-470-27858-1, Hoboken, New Jersey

Weigdong, J.; Wang, J. \& Zhang, J. (2014). An improved real hybrid genetic algorithm. Technical Gazette, Vol. 21, No. 5, pp. 979-986, ISSN 1330-3651

Wikipedia ${ }^{1}$. Convolutional neural network, available from: https://en.wikipedia.org/ wiki/Convolutional_neural_network. Accessed: 2020-07-10

Wikipedia $^{2}$. Evolucijski algoritem, available from: https://sl.wikipedia.org/ wiki/Evolucijski_algoritem. Accessed: 2020-07-10

Wikipedia ${ }^{3}$. Genetic algorithm, available from: https://en.wikipedia.org/ wiki/Genetic_algorithm. Accessed: 2020-07-22

Zhang H. \& Zhang Y. Q. (2020). A discrete job-shop scheduling algorithm based on improved genetic algorithm. International Journal of Simulation Modelling, Vol. 19, No. 3, pp. 517-528, ISSN 1726-4529 